\title{
Evaluación de tecnologías de red inalámbrica para Aplicaciones domóticas e inmóticas (edificios inteligentes). ${ }^{1}$ Evaluation of Wireless Network Technologies for Domotic and Inmotic Aplications (Smart Buildings).
}

\author{
Luis Alejandro Osorio Amaya ${ }^{2}$, Sergio Andrés Zabala Vargas ${ }^{3}$, Tatiana Inés Navas Gómez \\ ${ }^{1}$ Corporación Universitaria de Investigación y Desarrollo, Colombia.
}

Artículo recibido en mes Enero de 2013; artículo aceptado en mes mayo de 2013

\begin{abstract}
Citación del artículo: Osorio, L., Zabala, S. \& Navas, T. (2013). Evaluación de tecnologías de red inalámbrica para aplicaciones domóticas e inmóticas (edificios inteligentes). $I+D$ Revista de Investigaciones, 1(1), 6-15.
\end{abstract}

\section{Resumen}

La implementación de sistemas domóticos/inmóticos es costosa en general, debido a la necesidad de acondicionamiento de los nuevos esquemas de comunicación, cableado y tuberías en las instalaciones objetivo. En la actualidad se encuentran disponibles tecnologías populares de comunicación inalámbrica que podrían ser útiles para resolver este problema tanto por sus ventajas de costo-eficiencia como por su costo-efectividad. Se plantea un estudio tecnológico aplicado, de nivel evaluativo, que permita establecer cuáles son las tecnologías que cumplen estas condiciones y que pueden ser aplicadas ulteriormente en la planta física de la sede de la Corporación Universitaria de Investigación y Desarrollo (UDI) en la ciudad de Bucaramanga (Colombia).

Palabras clave: Domótica, inmótica, Wi-Fi, ZigBee, Bluetooth, Arduino, RabbitCore.

${ }^{1}$ Artículo de investigación tecnológica, de enfoque cuantitativo, resultado parcial de un proyecto de investigación en desarrollo en el grupo de investigación en Robótica, Control y Procesamiento de Señal GPS (categoría "A" de COLCIENCIAS) y fue financiado por la Corporación Universitaria de Investigación y Desarrollo (UDI) de la ciudad de Bucaramanga (Colombia). Calle 9 No. 23-55, PBX: 6352525.

${ }^{2}$ Pregrado en Tecnología, Unidades Tecnológicas de Santander; Ingeniero Electrónico, Universidad del Valle. DocenteInvestigador del grupo: Robótica, Control y Procesamiento de Señal GPS. Corporación Universitaria de Investigación y Desarrollo (UDI) de la ciudad de Bucaramanga (Colombia): Calle 9 No. 23-55, PBX: 6352525. Correo electrónico: luis.osorio@udi.edu.co

${ }^{3}$ Ingeniero Electrónico, Universidad Industrial de Santander; Especialista en Gerencia de Proyectos, Universidad del Tolima. Docente-Investigador del grupo: Robótica, Control y Procesamiento de Señal GPS. Corporación Universitaria de Investigación y Desarrollo (UDI) de la ciudad de Bucaramanga (Colombia): Calle 9 No. 23-55, PBX: 6352525. Correo electrónico: sergiozabala@udi.edu.co

${ }^{4}$ Ingeniera Electrónica, Universidad Industrial de Santander; Especialista en Telecomunicaciones, Universidad Industrial de Santander. Docente-Investigadora del grupo: Robótica, Control y Procesamiento de Señal GPS. Corporación Universitaria de Investigación y Desarrollo (UDI) de la ciudad de Bucaramanga (Colombia): Calle 9 No. 23-55, PBX: 6352525. 


\section{Abstract}

Implementation of domotic and inmotic systems is generally costly, because of the conditioning of new communication ways, wiring and piping within the target facilities. There are several popular technologies available at current time, which could be useful to solve this issue, both for its cost-efficiency and for its cost-effectiveness advantages. This paper proposes an evaluative-applied-technological study in order to establish which are the technologies that meet the aforementioned conditions to be applied further at the Corporación Universitaria de Investigación y Desarrollo (UDI) buildings in Bucaramanga (Colombia).

Keywords: Domotic, inmotic, Wi-Fi, ZigBee, Bluetooth, Arduino, RabbitCore.

\section{Introducción}

En la sede de la Corporación Universitaria de Investigación y Desarrollo (UDI) de la ciudad de Bucaramanga, no existe en la actualidad un sistema de control centralizado y automatizado que permita darle la denominación de edificio inteligente a los bloques que conforman su planta física. Las razones pueden ser varias, siendo la más importante, la falta de trabajos de investigación que apunten a desarrollar a mediano plazo proyectos de este tipo, iniciando desde lo general hacia lo particular en términos tecnológicos y que generen soluciones en la misma proporción, para ofrecer valor agregado, con costos de implementación razonables.

De otro lado y muy a pesar de todas sus bondades, los sistemas domóticos/inmóticos no han penetrado el mercado colombiano masivamente, debido, básicamente, a sus altos costos para su puesta en marcha. A nivel mundial, se desarrollan trabajos que hacen uso de las comunicaciones inalámbricas como método de enlace entre unidades domóticas distribuidas en edificaciones, buscando evitar el uso de extensos cableados en locaciones, y reducir los costos de implementación de un sistema de este tipo (Tafazolli, 2006; Romero, Vázquez \& Castro, 2007).

Este trabajo realiza una revisión de algunas tecnologías inalámbricas de comunicación, de importancia en aplicaciones industriales y domésticas, para posteriormente hacer una exploración práctica que permita ver las posibilidades reales de implementación de éstas en un sistema domótico/inmótico creado a medida de la UDI, que permita la futura automatización de sus edificios con miras a una mejor prestación de sus servicios y una disminución en sus gastos operativos.

El estudio sigue el modelo de gestión en un sistema domótico/inmótico, mediante el cual se pretende disminuir notablemente los tiempos de respuesta ante cualquier situación en un edificio/industria y agilizar los procesos internos de asignación de recursos físicos. Estos son clasificados en cinco categorías: El de gestión de la energía, relacionada con la programación y zonificación de la climatización, el control automático de activación de cargas eléctricas, la gestión de tarifas eléctricas, la detección de apertura de ventanas y puertas; el de gestión de la seguridad, relacionado con la gestión del control de acceso con reconocimiento o identificación de los usuarios, control de presencia y detección de intrusos, detección de rotura de cristales y 
forzado de puertas, la video-vigilancia a través de cámaras, la seguridad de las personas y detección de averías de diverso tipo en instalaciones; el de gestión del confort, relacionada con la automatización de todos los distintos sistemas/instalaciones/equipos que se encuentren en el área de trabajo, dotándolos de control eficiente y de fácil manejo y supervisión, entre otros; el de la gestión de las comunicaciones, orientada a captar, transportar, almacenar, procesar y difundir datos o información por las diferentes áreas/zonas de interés (Tafazolli, 2006; Seel, 2007; Romero et al., 2007).

Por otra parte, se tiene en cuenta que existen diferentes redes que se encargan de manejar los sistemas de gestión mencionados anteriormente (subredes en sistemas domóticos/inmóticos: Red de datos, de seguridad, multimedia, red domótica), por lo tanto, se deben integrar estas distintas subredes físicas, para posibilitar la gestión y el control de manera centralizada, respondiendo a las necesidades de los usuarios que se encuentran en el espacio de influencia del sistema. Es aquí donde las tecnologías inalámbricas tienen un papel importante, toda vez que permiten la conexión de cada una de las redes mencionadas con el resto de los componentes del sistema, sin la necesidad de cableado y sus costos adicionales inherentes. Es importante, entonces, plantear una exploración de las capacidades de estas tecnologías para esbozar un diseño de red/redes de comunicación, un sistema domótico/inmótico a través de tres tecnologías, que, por sus características y viabilidad pueden ser: Dos tecnologías de red de área personal $(P A N)$ : Bluetooth (802.15.1) y ZigBee (802.15.4), y una tecnología de red WLAN: WiFi(802.11).
Con base en lo anterior, se planteó como objetivo del estudio evaluar las tres tecnologías principales de la comunicación inalámbrica, con énfasis en PAN (Bluetooth (802.15.1) y ZigBee (802.15.4), y una tecnología de red WLAN: WiFi (802.11) en su funcionalidad para aplicaciones domóticas costo-efectivas y costo-eficientes con ulterior utilización, para las instalaciones de la sede de la UDI.

\section{Método}

\section{Tipo de estudio}

Se siguió un tipo de investigación tecnológica aplicativa - en la cual la solución es el problema de nivel evaluativo, sobre la efectividad y el funcionamiento óptimo de los dispositivos objetivo de prueba: Las tres tecnologías de la comunicación inalámbrica con énfasis en PAN: (Bluetooth (802.15.1), ZigBee (802.15.4), y una tecnología de red WLAN: WiFi (802.11), mediante ensayos de prueba de laboratorio para cada dispositivo.

\section{Participantes}

Experimentador: docente del área y estudiantes.

\section{Materiales e instrumentos tecnología de bluetooth.}

(a) tarjeta Arduino Duemilanove ${ }^{\circledR}$ (ver figura 1) basada en un microcontrolador de 8 bit ATMega 328®, de hardware abierto; (b) la tarjeta Roving Networks RN41® para hacer prototipos, dispone de un puerto serial estándar para interconexión con cualquier dispositivo que también posea puerto serie ( $\mathrm{O}^{\prime}$ Sullivan \& Igoe, 2004; Igoe, 2007); (c) el Bluegiga Access Server 
$2293 \AA$ (ver figura 2), este access server es un router inalámbrico Bluetooth, versión 2.0 de este estándar, posee conectividad total con redes $T C P / I P{ }^{\circledR}$ (A7 Engineering Incorporated, 2005).

Figura 1. Tarjeta Arduino + RN41 SDK Bluetooth ${ }^{\circledR}$

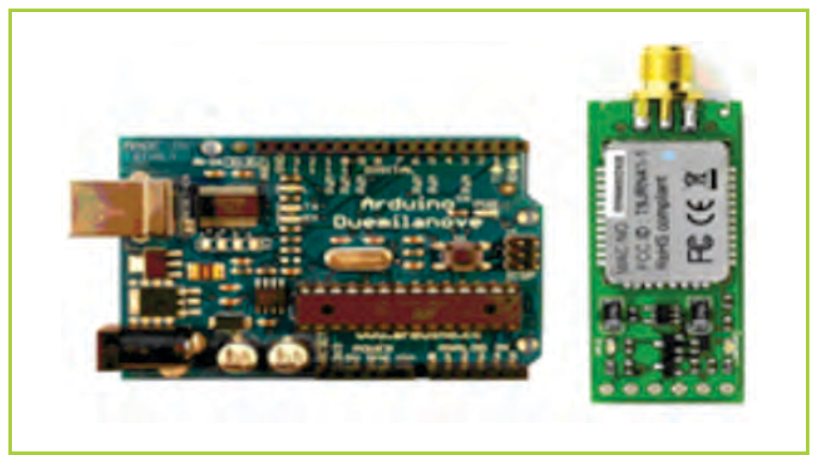

Fuente: Autor

Figura 2. Bluegiga Access Server 2293®

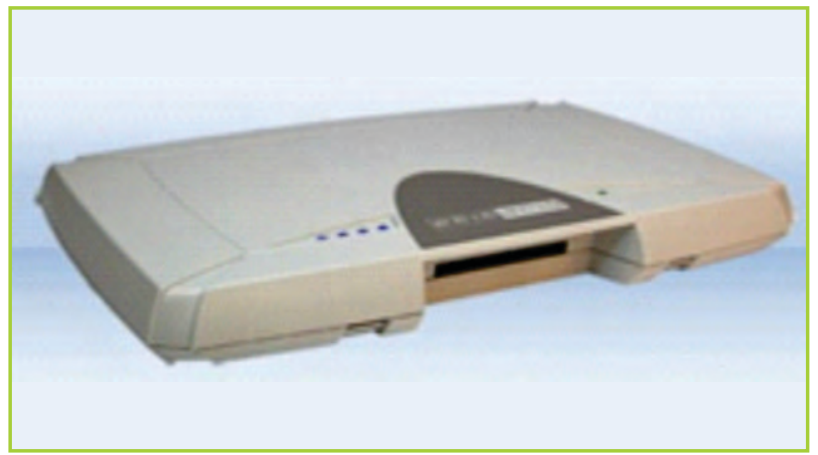

Fuente: A7 Engineering Inc.

\section{tecnología zigbee.}

(a) tarjeta Arduino Duemilanove ${ }^{\circledR}$ que se describió antes; (b) Xbee Shield ${ }^{\circledR}$ para Arduino (ver la figura 3), de hardware abierto, interacción con los radios ZigBee, llamados XBee (O'Sullivan \& Igoe, 2004; Igoe, 2007); (c) Módulo X4 Digi ${ }^{\circledR}$ que es un Gateway inalámbrico diseñado para conectar redes ZigBee/802.15.4 con TCP/IP (Digi International Incorporated, 2010; Payne, 2010).
Figura 3. Módulo XBee Shield $₫$ con la tarjeta Arduino

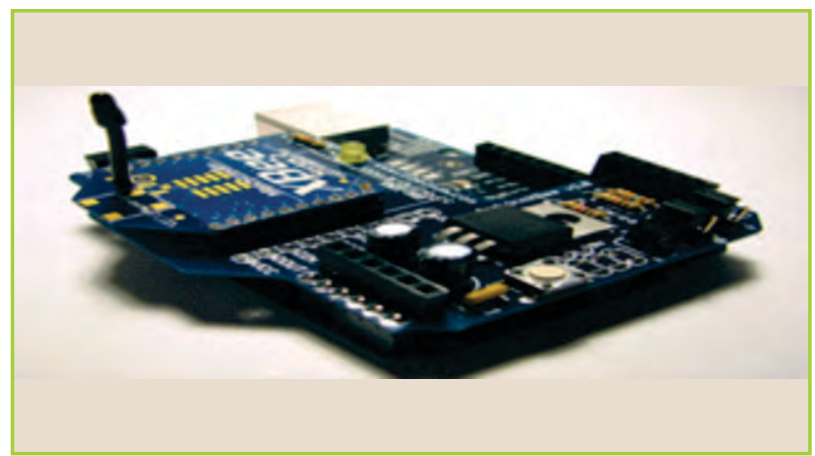

Fuente: Autor

\section{tecnología de wifi.}

(a) La tarjeta de desarrollo BL5S220® (ver figura 4) junto al módulo inalámbrico RCM5400W RabbitCore ${ }^{\circledR}$, manipula 40 puertos de entrada/salida, puertos analógicos/digitales con estándar 802.11 (Digi International Incorporated, 2008a, 2008b,2008c).

Figura 4. Tarjeta de desarrollo B15S220 de Rabbit/Digi ${ }^{\circledR}$



Fuente: Autor

\section{Procedimiento}

Se llevaron a cabo pruebas y ensayos de análisis para cada una de las tres tecnologías a través de diferentes prácticas de laboratorio, así: 


\section{usando tecnología bluetooth.}

Integración Arduino Duemilanove y módulo RN41® de Roving Networks $@$, transmisión de datos con tecnología Bluetooth entre dos tarjetas Arduino Duemilanove ${ }^{\circledR}$ utilizando módulo RN41 de Roving Networks ${ }^{C}$, sensado y transmisión de datos con tecnología Bluetooth entre dos tarjetas Arduino Duemilanove ${ }^{\circledR}$, visualización de datos transmitidos inalámbricamente con tecnología Bluetooth en un $L C D \AA$, enlace punto a punto bidireccional de monitoreo y alarma de respuesta con tecnología Bluetooth, administración del Access Server Bluegiga 2293® por interfaz Web.

\section{usando tecnología zigbee.}

Comunicación de dos tarjetas Arduino Duemilanove utilizando dos Xbee Shield® ${ }^{\circledR}$ y el programa $X-C T U \AA$, activación de un $L E D \circledR$ con la tecnología Zigbee implementando los módulos Xbee Shield ${ }^{\circledR}$ y las tarjetas de desarrollo Arduino Duemilanove ${ }^{\circledR}$, comunicación serial via X-CTU® y tarjeta Arduino Duemilanove $\AA+$ LCD $\AA$, mediante módulo Xbee Shield y tarjeta de desarrollo DIGI $P K G$-U; comunicación entre dos módulos Xbee Shield ${ }^{\circledR}$ con sus respectivas tarjetas de desarrollo Arduino Duemilanove ${ }^{\circledR}$ observando datos a través de un display LCD ${ }^{\circledR}$, lectura de temperatura (LM335) entre dos tarjetas Arduino Duemilanove ${ }^{\circledR}+\operatorname{LCD}{ }^{\circledR}$ y módulos Xbee Shield ${ }^{\circledR}$, lectura y transmisión de la variable humedad mediante módulos Xbee Shield® y tarjetas Arduino Duemilanove ${ }^{\circledR}$; configuración del Gateway X4® a través del programa Digi Device Discovery ${ }^{\circledR}$, medición del nivel RSSI ${ }^{\circledR}$ de los radios XBee ${ }^{\circledR}$ mediante la herramienta incorporada en el programa X-CTU®. Medición del alcance máximo de los módulos y su potencia de transmisión.

\section{usando tecnologia wifi.}

Lectura y modificación del estado de dos LED ${ }^{\circledR}$ en el módulo Rabbit Rcm5400w ${ }^{\circledR}$ conectado a la tarjeta prototipo Rabbit Demo Board $\AA$, cargar y leer VBAT-RAM ${ }^{\circledR}$ usando un indicador visual de detección, mediante tarjeta prototipo Rabbit Demo Board $\AA$, captura de un pulso PPM en la tarjeta BL5S220® usando como visualizador un osciloscopio; realización de un ping desde tarjeta BL5S220 $®$ a un $P C$ por $\mathrm{WiFi}{ }^{\circledR}$.

\section{Resultados}

La evaluación de las tres tecnologías objetivo se llevó a cabo a través de criterios de frecuencia, transferencia, canales, tipos de dato, alcance del dispositivo, número de nodos, complejidad, mercado, topología, aplicación, consumo de potencia promedio, tipos de datos y precio, encontrándose características ventajosas en cada caso (ver tabla 1).

Para la tecnología de Bluetooth, la tarjeta Arduino Duemilanove ${ }^{\circledR}$ mostró su buen funcionamiento bajo la filosofía de hardware abierto, tiene un bajo costo y desarrollo en línea de respaldo mundial. Las pruebas con la tarjeta Roving Networks RN41® mostraron buen desempeño para prototipos utilizando la tecnología Bluetooth; se le encuentran ventajas en que dispone de puerto serial estándar para interconexión con cualquier dispositivo que también posea puerto serie. Adicionalmente se probó la Bluegiga Access Server 2293®, un router inalámbrico Bluetooth que posee conectividad total con redes TCP/IP $®$; mostró en las pruebas capacidades para la centralización de 
Tabla 1. Características de las tecnologías: Bluetooth (802.15.1), ZigBee (802.15.4) y WLAN: WiFi (802.11).

\begin{tabular}{|c|c|c|c|}
\hline \multirow[t]{2}{*}{ Criterios de evaluación } & \multicolumn{3}{|c|}{ Tecnologías } \\
\hline & WiFi & Bluetooth & ZigBee \\
\hline Frecuencia & $2.4 \mathrm{GHz}$ & $2.4 \mathrm{GHz}$ & $2.4 \mathrm{GHz}$ \\
\hline & & & $868 / 915 \mathrm{Mhz}$ \\
\hline Transferencia & $101 \mathrm{Mbps}$ & $1 \mathrm{Mbps}$ & $250 \mathrm{kbps}(2.4 \mathrm{GHz})$ \\
\hline \multirow{5}{*}{ Canales } & & & 40kbps $(915 \mathrm{MHz})$ \\
\hline & $10-14$ & 79 & $20 \mathrm{kbps}(868 \mathrm{MHz})$ \\
\hline & & & $16(2.4 \mathrm{GHz})$ \\
\hline & & & 10 (915MHZ) \\
\hline & & & $1(868 \mathrm{MHz})$ \\
\hline Tipos de dato & Digital & Digital, Audio & Digital (Texto) \\
\hline Alcance de & $100 \mathrm{~m}$ & $10 m-100 m$ & $10 m-100 m$ \\
\hline dispositivos & 32 & 8 & $255 / 65535$ \\
\hline Número de nodos & Complejo & Complejo & Simple \\
\hline \multirow{2}{*}{ Complejidad } & Alta & Media & Baja \\
\hline & Estrella & Estrella & Estrella, Arbol, \\
\hline Mercado & & & Punto a Punto y Malla \\
\hline Topología & Alta necesidad de & Equipos móviles en & Control y monitorización \\
\hline Aplicación & datos & general & industrial \\
\hline $\begin{array}{c}\text { Consumo potencia } \\
\text { promedio }\end{array}$ & $400 \mathrm{~mA}$ & $40 \mathrm{~mA}$ & $40 \mathrm{~mA}$ \\
\hline Tipos de datos & Digital & Digital & Digital \\
\hline Precio & Costoso & Accesible & Accesible \\
\hline
\end{tabular}

Fuente: Autor

los servicios implementados en Bluetooth hacia internet; es un elemento clave para la convergencia de las redes y subredes dentro del sistema deseado.

De la tecnología ZigBee se probó la tarjeta Arduino Duemilanove, la Xbee Shield ${ }^{\circledR}$ para Arduino, que mostró perfecta interacción con los radios ZigBee®, el Módulo X4 para conectar redes $Z$ igBee/802.15.4® con TCP/IP ${ }^{\circledR}$; se observó facilitación de la convergenciaintegración para los elementos controlados / monitorizados por esta subred con instancias superiores de aplicación.

De la tecnología $\mathrm{WiFi}{ }^{\circledR}$ se probó la tarjeta de desarrollo BL5S220®, observando su desempeño junto al módulo inalámbrico RCM5400W RabbitCore ${ }^{\circledR}$, verificando su 
capacidad para manipular 40 puertos de entrada/salida de bajo y alto consumo de corriente, lo que amplía las posibilidades de control y convergencia en el sistema domótico/inmótico deseado.

\section{Discusión}

A partir de los resultados obtenidos en las pruebas de laboratorio, se midieron diversos aspectos de las tres tecnologías objetivo, constatando la utilidad, eficacia y eficiencia de las mismas para producir soluciones en comunicación inalámbrica. Para la tecnología de Bluetooth, la tarjeta Arduino Duemilanove mostró su buen funcionamiento, bajo costo y el soporte que le da una de las comunidades de desarrollo en línea más grandes del mundo (Evans, 2007; Banzi, 2009; Oxer \& Bleming, 2009). De manera similar, la tarjeta Roving Networks RN41® mostró ser apropiada para hacer prototipos utilizando la tecnología Bluetooth, con su facilidad para la interconexión con cualquier dispositivo que también posea puerto serie (O'Sullivan \& Igoe, 2004; Igoe, 2007). También se encontró capacidad para la conectividad con redes $\mathrm{TCP} / \mathrm{IP} \AA$, para la centralización de los servicios implementados en Bluetooth hacia internet (A7 Engineering Incorporated, 2005) y para la convergencia de redes y subredes, lo cual es un aspecto fundamental de las comunicaciones inalámbricas, como lo es la facilitación para la convergenciaintegración en el sistema domótico/inmótico deseado (Digi International Incorporated, 2008a, 2008b, 2008c), que se halló en los dispositivos de las tecnologías evaluadas en lo atinente al control y la monitorización (Digi International Incorporated, 2010; Payne, 2010).
Las tres tecnologías de red inalámbrica son ideales para aplicaciones en domótica, cada una tiene cualidades y aplicaciones distintas: Bluetooth, con toda la capacidad para realizar control y monitorización, pero sobre todo para brindar contenidos a la gran cantidad de dispositivos móviles que poseen los usuarios de las áreas de influencia de un sistema domótico/inmótico; Zigbee ${ }^{\circledR}$, como vía de comunicación para sistemas de control y monitorización en campo y aplicaciones donde el consumo de energía requerido sea realmente bajo; y WiFi ${ }^{\circledR}$, como canal de banda ancha por donde enviar y recibir información de gran tamaño, pero también como ruta para ejercer control directo sobre cargas eléctricas conectadas al sistema (a un costo muy superior que Bluetooth ${ }^{\circledR}$ o ZigBee () .

Un sistema domótico/inmótico se compone de subredes que atienden necesidades puntuales y de interés para cada sistema en particular; estas subredes son acopladas entre sí para lograr control centralizado sobre todos los servicios implementados. Se resalta la importancia del desarrollo de programas para los elementos de red integradores a redes principales de datos (Gateway®).

Bluetooth es, de las tecnologías $W P A N \AA$, la más difundida en todo el mundo, madura en aspectos técnicos (Huang \& Rudolph, 2007), documentación, desarrollo tecnológico y está soportada por cientos de empresas que adaptan esta forma de comunicación inalámbrica a miles de sus dispositivos. Estas características son el punto más fuerte que ofrece Bluetooth como una tecnología aplicable a desarrollos domóticos. 
Trabajar con la plataforma Arduino ha permitido reducir el costo tanto en tiempo de desarrollo como en costo de implementación de las prácticas realizadas con la tecnología Bluetooth y ZigBee ${ }^{\circledR}$. La tecnología ZigBee ${ }^{\circledR}$ tiene todas las cualidades para convertirse en la primera opción para cuando se requiera hacer control y monitorización de eventos en el sistema a implementar. Falta todavía mucho para que sea de uso masivo, así como lo es hoy el Bluetooth o WiFi, pero debido a sus grandes prestaciones se espera que en los próximos años esta brecha sea cada vez menor y su acogida obligue a una baja en los costos de estos equipos.

\section{Referencias}

A7 Engineering Incorporated (2005). Embedded Blue 506. User Manual. Recuperado de:

http://www.xilinx.com/support/documentation/ boards_and_kits/ug349.pdf

Banzi,M. (2008). Getting Started with Arduino. Make: Projects.

Digi International Incorporated. (2008a). XCTU Configuration \& Test Utility Software. Recuperado de:

http://www.kikiwi.fr/uploads/data/XBEE/xctu manual_a.pdf

Digi International Incorporated. (2008b). BL4s200. C-Programmable Single-Board Computer with Networking. User's Manual. Recuperado de: http://ftp1.digi.com/support/documentation/019 -0171_F.pdf
Digi International Incorporated. (2008c). Dynamic C. Integrated C Development System for Rabbit 4000 and 5000. Recuperado de :

http://cholla.mmto.org/computers/rabbit/DynC FunRef.pdf

Digi International Incorporated. (2010). ConnectPort X Family User's Guide. Recuperado de:

http://dlnmh9ip6v2uc.cloudfront.net/datasheets /Wireless/Zigbee/90000832_E.pdf

Evans, B. (2007). Arduino Programming Notebook. Recuperado de:

http://playground.arduino.cc/uploads/Main/ard uino_notebook_v1-1.pdf

Huang, A. \& Rudolph, L. (2007). Bluetooth essentials for programmers. Massachussetts Institute of Technology. New York: Cambridge University Press.

Igoe, T. (2007). Making Things Talk. Practical Methods for Connecting Physical Objects. Maker Media, Inc.

O'Sullivan, O. \& Igoe, T. (2004). Physical Computing. Sensing and controlling the physical world with computers. Thomson, 2004.

Oxer, J. \& Blemings, H. (2009). Practical Arduino. Cool projects for Open Source Hardware. Springer Verlag GmbH.

Payne, J. (Ed) (2010). Beginning Python. Using Python 2.6 and Python 3.1. Wrox. 2010

Romero, C., Vázquez, F. \& Castro, C. (2007). Domótica e Inmótica - Viviendas y 
Edificios Inteligentes. España: Editorial Alfaomega.

Seel, N. (2007). Business Strategies for the next generation network. New York: Auerbach Publications.
Tafazolli, R. (Ed) (2006). Technologies for the wireless future. Vol. 2. Wireless World Research Forum (WWRF). England: John Wiley \& Sons, Ltd.

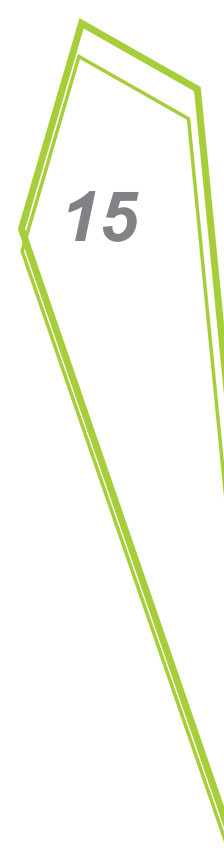

